\title{
Reviewer's comment concerning "CT morphometry of adult thoracic intervertebral discs" by Justin G. R. Fletcher et al. (Eur Spine J, 2015; doi:10.1007/s00586-015-3925-y)
}

\author{
René Schmidt $^{1}$
}

Received: 14 May 2015/Revised: 14 May 2015/Accepted: 14 May 2015/Published online: 21 May 2015

(C) Springer-Verlag Berlin Heidelberg 2015

The authors present a CT-based study on thoracic disc dimensions in deceased persons. Their conclusion is that their study "provides unique reference data on adult thoracic intervertebral disc morphometry". Despite the effect that it is easy to measure a static rigid subject such as the bone dimensions in this study, it is much more difficult for a mobile changeable thing as the disc. Surely it is easy to measure anterior or posterior disc height, but the results will be probably significantly different as a result of the position before the measurement. Magnusson et al. [1] already found a decrease of disc height while standing after longer lying before in different age groups. And although these data were obtained indirectly by body height, it is clear that body posture has an influence. For reference data one would claim that a definition in which position and after which prearrangement the measurement should be performed exists or at least one should provide the difference between two positions. So it might be that dimensions here are bigger due to the longer lying before the measurement. On the other hand, we are not aware whether in deceased persons the increase of disc height in an unloaded state still occurs or not. Summarizing, concerning the disc height and derived calculations reference data without definition cannot be obtained. Consecutively "interpreting pathological changes" in the clinical situation with this data should remember this drawback.

Conflict of interest None.

\section{Reference}

1. Magnusson M, Hult E, Lindström I, Lindell V, Pope M, Hansson T (1990) Measurement of time-dependent height loss during sitting. Clin Biomech 5:137-142

René Schmidt

rene.schmidt@af-k.de

1 Department of Orthopedics and Traumatology, Alb Fils Hospital, Eichertstr. 3, 73035 Göppingen, Germany 